\title{
Review on Vehicle Detection Based on Video Processing
}

\author{
Jiao Zhiyuan, Xing Yanfeng \\ Automobile Engineering College, Shanghai University Engineering Science, Shanghai, China \\ Email address: \\ 593511032@qq.com (Jiao Zhiyuan),xyf2001721@sues.edu.cn (Xing Yanfeng)
}

To cite this article:

Jiao Zhiyuan, Xing Yanfeng. Review on Vehicle Detection Based on Video Processing. International Journal of Science, Technology and Society. Vol. 5, No. 4, 2017, pp. 126-130. doi: 10.11648/j.ijsts.20170504.21

Received: May 19, 2017; Accepted: June 2, 2017; Published: July 18, 2017

\begin{abstract}
Compared with traditional vehicle detectors, video sensor has lots of advantages, i.e., easy installation and maintenance, wide monitoring areas, obtaining more kinds of traffic parameters and etc, so it has been widely used in Intelligent Traffic Systems. On this basis, discuss about the vehicle detection methods based on feature, model checking, frame difference, optical flow field. At the same time, the verification method is introduced, and the advantages and disadvantages of various algorithms are analyzed and compared. Finally, some suggestions for future research and application are presented, for example, vehicle detection is carried out by using a variety of detection methods and multi detector information fusion.
\end{abstract}

Keywords: Intelligent Transportation System, Vehicle Detection, Monocular Vision

\section{Introduction}

In recent years, with the rapid development of transportation, the research and application of intelligent transportation system have been paid more and more attention to. Intelligent transportation system is an advanced information technology, data communication transmission technology, electronic sensor technology, electronic control technology and computer applied to the whole integrated tube processing technology, and effectively established in a wide range, real-time, accurate and efficient integrated transport management system [1].

To realize the intelligent transportation system, the detection and tracking of vehicles are essential. The detection of vehicles is the premise of the whole process. The vehicle identification and detection technology is the generated under such demand.

At present, the commonly used vehicle detection methods are: loop magnetic induction coil detection, ultrasonic testing, microwave radar detection, infrared detection, pneumatic pipe inspection, photoelectric detection, video based vehicle detection and so on. Video detection is a branch of computer vision application. It is a combination of video image and computer pattern recognition. Video camera and computer imitate the function of human eyes, and video detection technology is becoming the most advantage and the most potential detection method in traffic monitoring system.

\section{Video Based Vehicle Detection Algorithm}

Video based vehicle detection algorithms can be divided into the following categories: feature detection, model-based detection, frame based detection, optical flow detection, and the following categories are introduced respectively.

\subsection{Feature Detection}

A feature-based approach is also known as a knowledge-based approach. Symmetry [2], shadow [3], edge [4] and so on are the common characteristics of vehicle detection. The brightness value of the shadow area at the bottom of the vehicle is obviously different from other parts of the image under better lighting conditions in the daytime. Using the symmetry of the vehicle, the position of the vehicle can be obtained from the symmetry map. Using the characteristics of the vehicle edge, the vehicle in the image can be segmented well, as shown in Figure 1. The edge feature is stronger than the shadow and symmetry feature. Shadows and symmetries are generally used as auxiliary features, and shadow, symmetry and edge features are used together, so that better detection results can be obtained $[5,6]$. 


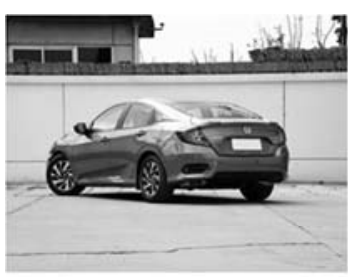

(a) Original

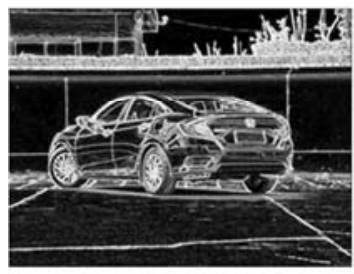

(c) Scharr filtering map

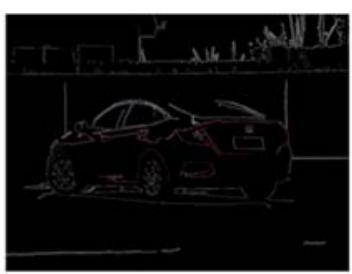

(b) Canny edge detection

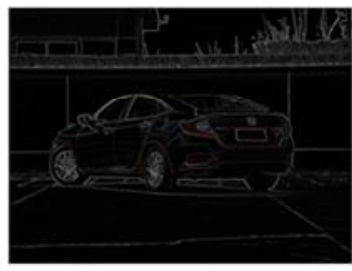

(d) Sobel edge detection
Figure 1. Edge detection.

\subsection{Model Based Detection}

Model based methods usually obtain a large number of different road environment and different types of vehicle images, and then use the gray information method to build the vehicle feature model. These models are matched with the image to be identified to find the characteristic areas similar to the model, i.e., the vehicles to be detected. In practical applications, the region of interest is first created by feature based method and then matched to improve the matching speed.

The establishment of an appropriate model is a prerequisite for this approach to understand the content of the image, and the pre-established model usually includes a camera model and a vehicle model. Because of the fixity of model matching, the system must set a template for each type of vehicle. This has some problems because even vehicles belonging to the same class may still have different geometries. At the same time, template methods are usually not change vehicle brightness characteristics hypothesis, but in reality, differences in the characteristics of environment illumination, shadow and vehicle body and window overlap and light reflection may be caused by the same type of vehicle, using the deformable model segmentation and recognition of vehicle to solve vehicle shape difference problems [7, 8].

Due to the over dependence of vehicle model based on the model method, a model can not be applied to all vehicles because of the diversity of vehicles. The change of vehicle attitude also makes the fixed model difficult to adapt. A fine model potential is built for each vehicle and gesture, which results in the increase of computational complexity. It is not beneficial to real-time processing when $3 \mathrm{D}$ model is adopted.

\subsection{Frame Difference Detection}

The frame difference method is to subtract the gray values of the pixels corresponding to the previous and two frames. If the gray difference is very small, the point does not pass through the vehicle; on the contrary, it is considered that the vehicle has passed. The frame difference method is characterized by simple implementation, fast operation, strong adaptability to the dynamic environment and insensitivity to changes in light. When using this method, it is necessary to consider how to select the appropriate time interval for differential, which generally depends on the speed of the monitored vehicle.

Frame difference method is based on the premise that the background is fixed. If the background changes, the method will fail. In this case, the neighborhood of two adjacent comparison combined with frame difference methods, to compensate for the background caused by camera motion movement, then the differential calculation, to overcome the traditional frame difference method in background removal of the phenomenon is not clean. Moving target detection based on symmetry difference operation, with 3 consecutive frames of image sequences for a set of advanced processing object, absolute difference operation, and automatic threshold filtering and maximum entropy segmentation to get the two value image, which makes the slow motion of two of the target pixel value segmentation algorithm can ensure the regional expansion good detection in complex background and low SNR and small moving target.

The inter frame difference method is a differential operation of two adjacent frames in a video sequence, in order to obtain the contour of the moving object, and has good applicability in the case of multiple moving objects and cameras moving [9]. The frame difference method is divided into two adjacent frames, the difference and the multi frame phase difference method.

\subsubsection{Adjacent Two Frame Difference Method}

When the moving object is abnormal in the monitoring scene, the difference between frames will be more obvious, the subtraction of two frames, two frames of image brightness difference absolute value, and then the threshold contrast analysis of the movement characteristics of video or image sequence, determine whether the objects in the image sequence [10].

The input frame at $t_{1}$ is $f_{1}(x, y)$, and the input frame at $t_{2}$ is $f_{2}(x, y)$. If there is a vehicle passing through this period, there is a $f_{1}(x, y)=f_{2}(x-\Delta x, y-\Delta y)$ :

$$
\Delta f(x, y)=\left|f_{2}(x, y)-f_{1}(x, y)\right|
$$

For the static part of the image, the $\Delta x=\Delta y=0$ is then $\Delta f(x, y)=0$, and the moving part $\Delta f(x, y) \neq 0$ is obtained so that the moving region of the vehicle is obtained. In the actual application, make difference of two adjacent frames, the segmentation by two value region is actually two position after the merger of the object and area larger than the actual, so the moving target internal prone to cavitation. Second, the algorithm detects $t$ and $t-\Delta t$ moments, which involves sampling frequency problems.

\subsubsection{Multi Frame Difference Method}

In order to solve the problem of the difference between adjacent frames, a multi frame difference method is proposed. 
In the image captured by the camera, three consecutive frames or frames are used as the 22 differences. The sequence of two valued images is $\left\{I_{m}\right\}$, and the image $D_{m}$ is defined as:

$$
D_{m}(i, j)=\left|I_{m}(i, j)-I_{m-1}(i, j)\right|
$$

Logical results can be obtained by subtracting multiple two valued image logic:

$$
D_{n, T}(i, j)=\left\{\begin{array}{l}
1, D_{n-1, T}(i, j)=1, \quad D_{n, T}(i, j)=1 \\
0, \text { else }
\end{array}\right.
$$

Where $T$ is the threshold, based on frame difference analysis method shows that the algorithm is simple, it has the advantages of low complexity of program design and calculation speed, and the change of light and other scenes of low sensitivity to a variety of dynamic environment adaptability, stability of ideal.

Frame difference method is simple and not easy to be affected by the change of environment light, but it can not detect stationary vehicle, and the processing effect is related to the sampling frequency of the image and the speed of the vehicle being detected. If the video detector sampling frequency is too small, and faster speed, may cause incorrect segmentation; if the sampling frequency is too large and the speed is slow, and it will cause excessive coverage, moving objects under extreme conditions may overlap, similar to a stationary vehicle, which lead to the segmentation of moving objects. In order to solve these problems, Kameda uses probabilistic statistical method to find ROI by moving information between three consecutive images.

\subsection{Detection Based on Optical Flow}

The basic idea of the method of optical flow field in the space, the movement can be described by the sports field, and in an image plane, the movement of objects is often through different reflect the different image gray distribution of image sequences, and space motion field image transferred to said optical flow field. The optical flow reflects the change trend of each gray image, can be regarded as the instantaneous velocity field of pixels with gray motion in the image plane and is also a kind of true motion field approximation.

Optical flow refers to the speed of mode motion in an image. The optical flow field is a two-dimensional instantaneous velocity field in which the two-dimensional velocity vector is the projection of the $3 \mathrm{D}$ velocity vector of the visible image on the imaging plane. Optical flow is a vector field that transforms the image of the detected region into a velocity. Each vector represents the instantaneous change of the position of a point in the image. Therefore, the optical flow field carries abundant information about the 3D structure of the object motion and the scene. Through the analysis of the velocity field, the vehicle in the detection area can be determined (Figure 2).

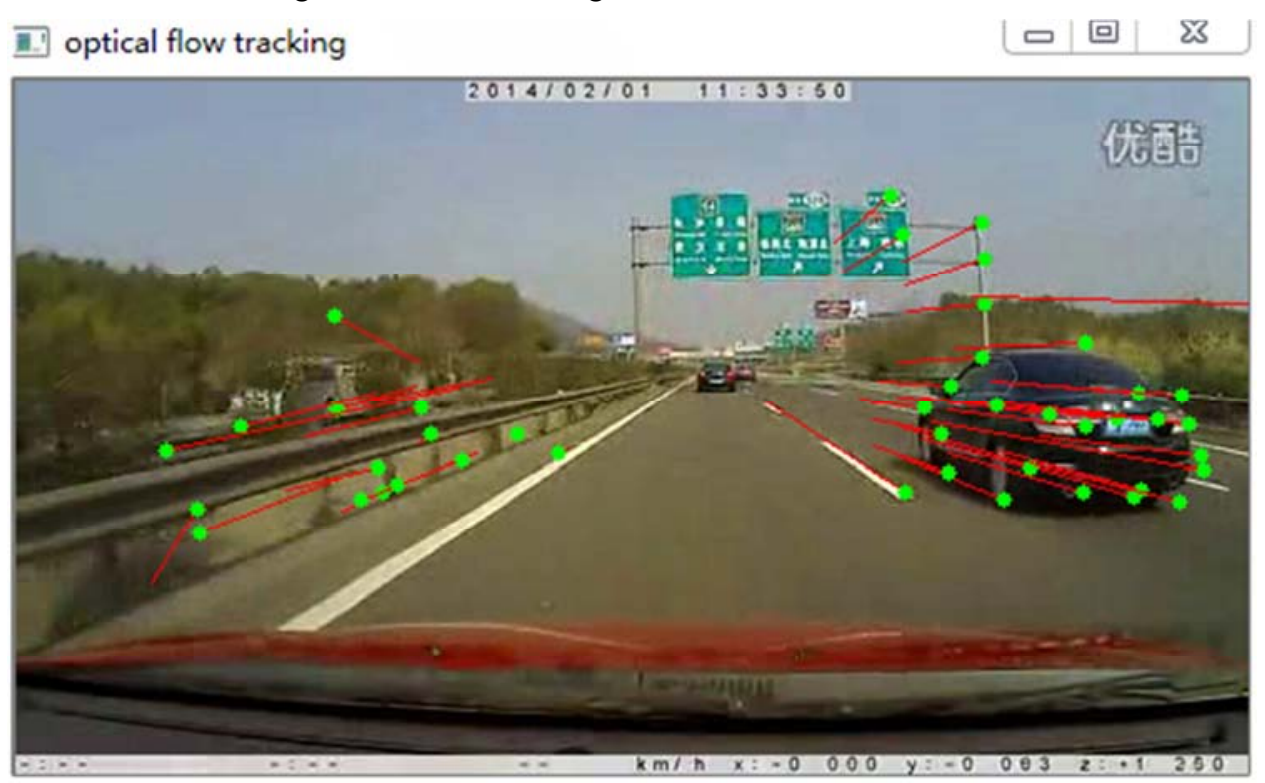

Figure 2. Optical flow detection.

Optical flow calculation methods are divided into: differential method [11], matching method [12], energy based method[13], phase based method[14] and so on.

Among them, differential method is the earliest and most widely used method, also known as the space-time gradient method. The basic principle is as follows: $I(x, y, t)$ is the gray level of the image point $(x, y)$ at $t$, and the $\frac{d_{x}}{d_{\mathrm{t}}}=u$, $\frac{d_{y}}{d_{t}}=v$ are the two speed component of the $(x, y)$ point at image detection area in the $x$ direction and the $y$ direction, that is, the required velocity vector $\mathrm{M}$. The gradient constraint equation of optical flow vector is derived according to the hypothesis of image gray maintenance:

$$
I_{x} u+I_{y} v+I_{t}=0
$$


Among them, $I_{x}, I_{y}$ and $I_{t}$ are partial derivatives of $I(x, y, t)$ in 3 directions along $x, y$ and $t$ respectively. By combining the gradient constraint equations with the overall smoothness constraints of the velocity field, the variational problem of $u$ and $v$ calculations is obtained:

$$
\left.\min \left\{\iint\left(I_{x} u+I_{y} v+I_{t}\right)+\left.\alpha^{2}|| \frac{d_{u}}{d_{x}}\right|^{2}+\left|\frac{d_{u}}{d_{y}}\right|^{2}+\left|\frac{d_{v}}{d_{x}}\right|^{2}+\left|\frac{d_{v}}{d_{y}}\right|^{2} \mid\right)\right\}
$$

Among them, $\left|\frac{d_{u}}{d_{x}}\right|^{2}+\left|\frac{d_{u}}{d_{y}}\right|^{2}$ is the Laplasse operator of $u$, $\left|\frac{d_{v}}{d_{x}}\right|^{2}+\left|\frac{d_{v}}{d_{y}}\right|^{2}$ is the Laplasse operator of $v$, and $\alpha^{2}$ is weight. For the Euler Lagrange equation above, initial values are set, and the velocity vectors of $(x, y)$ point satisfying some error are obtained after many iterations. The same method is used to obtain the velocity field of the whole image detection region, and the image can be analyzed according to the velocity vector feature of each pixel point. If no car through the static background, then optical flow vector changes continuously in the detection area; when a car passes, optical flow vector and optical flow vector is different the background, so as to detect the position of the vehicle and the.

Under ideal conditions, it can detect object in the movement, does not need to know any information of the scene and can accurately calculate the speed of moving objects, and can be used for the motion of the camera. But the optical flow method has the following disadvantages: sometimes even without motion, the external lighting changes, but also can be observed in the absence of optical flow; gray scale change enough area, the actual movement is often not observed. The brightness changes of $3 \mathrm{D}$ object motion is projected into $2 \mathrm{D}$ images, due to the loss of information itself and the optical flow method existence aperture problem and occlusion problem, using optical flow method to estimate the 2D motion field is uncertain, need to assume additional model to simulate the structure of 2D motion field; in accurate segmentation, optical flow method is needed to improve the accuracy of segmentation using the spatial features of color, gray, edge; at the same time as the iterative method was used to calculate the optical flow method, complicated and time-consuming, if there is no special hardware support, it is difficult to be used for real-time detection of video sequences.

\section{Conclusion}

The key to vehicle detection is to separate the vehicle from the background, which requires the use of some spatial or temporal features of different vehicle backgrounds. From the current detection methods, feature based and model based methods are more successful for detecting vehicles ahead. Optical flow method is more suitable for detecting vehicles passing from side to side. The key of feature based method is to find stable and reliable vehicle characteristics, and the fusion algorithm of multiple features is a way to improve the detection accuracy. The establishment of the correct vehicle model is the basis of model-based method. The fixed model can not adapt to the change of vehicle attitude, and the establishment of adaptive model algorithm and 3D model is the future research direction.

Compared with traditional detectors, video detectors have obvious advantages. They have been used more and more widely in intelligent transportation systems in recent years. Although video detectors have many advantages, there are still many problems that need to be solved. A traffic detection system based on video images can be put into practical use. It should have the characteristics of short processing time, low computation and high reliability. Moreover, the method adopted in this system must be robust enough to reconstruct the error of $3 \mathrm{D}$ scene, the image noise caused by vehicle movement and the offset of video detector. The main problems are: one is the detection accuracy of the video detector varies as the light changes, when the light is good, such as the detection accuracy of the best at midday and evening, such as rain and snow weather is poor; another problem is the problem of shadow, the shadow is mainly caused by the video detection method of error detection the shadow, usually has three kinds: the vehicles moving shadow, road scene in static shadow, slowly moving shadows of clouds in the shadows caused by occlusion; while the vehicle on the road in the scene, but also must be considered.

The future development in this field should be solved around the above issues, the main trend is: first, pay attention to the accuracy, the integrated use of multiple detection method is a development trend of future vehicle detection; in addition, multi detector information fusion in vehicle detection is the focus of future research; at the same time, the related research fields close contact with the video based vehicle detection, traffic video image compression and multimedia data mining, which is also the hotspot of future research.

\section{References}

[1] M Tomizuka. Automated Highway Systems: An Intelligent Transportation System for the Next Century [J]. IEEE International Symposium on Industrial Electronics, 1997, (1): 1-4.

[2] LIU T, ZHENG N, ZHAO L. Learning based Symmetric Features Selection for Vehicle Detection [C]. IEEE Proceedings of Intelligent Vehicles Symposium. IEEE, 2005: 124-129.

[3] CHARKARI N M, MORI H. A New Approach for Real Time Moving Vehicle Detection [C] Proceedings of the IEEE RSJ International Conference on Intelligent Robots and Systems. 1993, 1: 273- 278 .

[4] KUT SUMA Y, YA GUCHI H, HAMA MOTO T. Real time Lane Line and Forward Vehicle Detection by Smart Image Sensor [C]. IEEE International Symposium on Communications and Information Technology. IEEE, 2004, 2: 957- 962.

[5] HOFFMAN C, DANG T, STILLER C. Vehicle detection fusing 2D visual features [C]. IEEE Proceedings of Intelligent Vehicles Symposium. IEEE, 004: 280-285. 
[6] CLADY X, COLLANGE F, JURIE F. Cars detection and tracking with a vision sensor [C]. IEEE Proceedings of Intelligent Vehicles Symposium. IEEE, 2003: 593- 598.

[7] M P Dubuisson, S Lakshmanan, A K Jain. Vehicle Segmentation and Classification Using Deformable Templates [J]. IEEE Transactions on Pattern Analysis and Machine Intelligence, 1996, 18 (3): 293-308.

[8] J Ferryman, A Worrall. A Generic Deformable Model for Vehicle Recognition [C]. Proceedings of British Machine Vision Conference, 1995. 127-136.

[9] ABUTALEB A S. Automatic Thresh holding of Gray level Pictures Using Two-dimensional Entropy [J]. Computer Vision Graphics and Image Processing, 1989, 47 (2): 22- 32.
[10] PARKY. Shapere solving Local Thresh holding for Object Detection [J]. Pattern Recognition Letters, 2001, 22 (5): 883890.

[11] HORN BK P, SCHUNCK BG. Determining optical flow [J]. Artificial Intelligence, 1981, 17 (1): 185- 203.

[12] SINGH A, ALLEN P. Image flow computation: an estimation theoretic framework and a unified perspective [C]. Proc of CVG IP: Image Understanding. 1992, 56: 152- 177.

[13] HEEGER D J. Model for the extraction of image flow [J]. J Opt Soc Am, 1987 (4): 1455- 1471.

[14] FLEETDJ, JESPONAD. Computation of component image velocity from local phase information [J]. International Journal of Computer Vision, 1990, 5 (1): 77- 104. 